Témoigner Témoigner. Entre histoire et mémoire

Getuigen Revue pluridisciplinaire de la Fondation Auschwitz

$115 \mid 2013$

L'Espagne en construction mémorielle

\title{
Éric Alary et Bénédicte Vergez-Chaignon, Dictionnaire de la France sous l'Occupation
}

Léon Strauss

\section{(2) OpenEdition}

1 Journals

Édition électronique

URL : http://journals.openedition.org/temoigner/617

DOI : 10.4000/temoigner.617

ISSN : 2506-6390

Éditeur :

Éditions du Centre d'études et de documentation Mémoire d'Auschwitz, Éditions Kimé

Édition imprimée

Date de publication : 1 mars 2013

Pagination : 166-168

ISBN : 978-2-84174-628-6

ISSN : 2031-4183

Référence électronique

Léon Strauss, "Éric Alary et Bénédicte Vergez-Chaignon, Dictionnaire de la France sous l'Occupation », Témoigner. Entre histoire et mémoire [En ligne], 115 | 2013, mis en ligne le 01 juin 2015, consulté le 23 octobre 2020. URL : http://journals.openedition.org/temoigner/617 ; DOI : https://doi.org/10.4000/ temoigner.617 


\section{LIBRAIRIE}

Dictionnaire de la France sous l'Occupation

de Éric Alary et

Bénédicte Vergez-Chaignon

Compte-rendu par Léon Strauss

\section{PAGE 166}

Dictionnaire de la violence de Michela Marzano

Compte-rendu par Frediano Sessi

\section{PAGE 168}

Le Livre de Joseph

de Bernard Dan

Compte-rendu par Albert Mingelgrün

\section{PAGE 169}

The Sign of the Cross.

From Golgotha to Genocide de Daniel Rancour-Laferriere

Jésus de Nazareth. Tome 2 : De l'entrée à Jérusalem à la Résurrection de Joseph Ratzinger

Compte-rendu par Frans Lemaire

\section{PAGE 170}

Tiempo pasado

de Beatriz Sarlo

Compte-rendu par Mylène Herry

PAGE 176

Le Piège des loups.

Les 175 maisons

de la Gestapo en France

de Dominique Sigaud

Compte-rendu

par Corinne Benestroff

PAGE 179
Le Mythe du grand silence.

Auschwitz, les Français,

la mémoire

de François Azouvi

Compte-rendu

par Paul Bernard-Nouraud

PAGE 180

Triangle rose

de Michel Dufranne

Compte-rendu par Anthony Michel

\section{PAGE 186}

Qui écrira notre histoire ?

Les Archives secrètes du ghetto de Varsovie

de Samuel D. Kassow

Compte-rendu par Judith Lindenberg

PAGE 188

Une Éthique du regard

de Sylvie Rollet.

Compte-rendu

par Fabian Van Samang

PAGE 192

Aharon Appelfeld.

Je suis une rémanence de l'histoire juive

Compte-rendu par Julie Laval

PAGE 196 


\title{
Dictionnaire de la France sous l'Occupation
}

\author{
Éric Alary et Bénédicte Vergez-Chaignon, Paris, Larousse (Coll. À présent), 2011, 447 p.
}

Par Léon Strauss, Université de Strasbourg

Dans une collection destinée au grand public et aux étudiants, deux historiens spécialistes de la France de 1939 à 1945 présentent sous une forme assez succincte l'état actuel des connaissances sur la France de Vichy et de l'Occupation. Leur ouvrage est divisé en trois parties : la première « L'Occupation en questions » présente onze questions qui de 1940 à nos jours, des responsabilités de la défaite de 1940 à la survie du général de Gaulle dans le champ de la mémoire nationale ont préoccupé et/ou divisé l'opinion française et aussi la littérature politique et historique. Ensuite, « Les temps forts » présentent les grandes phases de la période : 1939, l'engrenage vers la guerre ; 1940 : les conséquences de la défaite et les premières dissidences ; 1940-1942 : un chapitre sur la collaboration d'État et le collaborationnisme, un autre sur la naissance de la Résistance ; le tournant relatif de novembre 1942 ; 1943-1944 : un chapitre consacré à l'insécurité annonciatrice de la guerre civile, un second à la bataille pour la légitimité menée par de Gaulle et la Résistance ; 1945 : la Libération et la victoire ; le dernier chapitre présente les soubresauts de la mémoire de 1944 à nos jours. Le dictionnaire proprement dit ne commence quà la $103^{\mathrm{e}}$ page. Les entrées parfois très sommaires (comme Alsace-Lorraine qui sous-estime la part du ralliement initial au nazisme dans ces régions annexées de fait), parfois relativement développées (Hitler et la France) sont très variées et couvrent la plupart des champs de l'historiographie. On sera particulièrement attiré par les articles qui résument les travaux antérieurs des deux auteurs. Ils avaient publié ensemble avec Gilles Gauvain en 2006 un ouvrage sur la vie quotidienne des Français de 1939 à 1949 : on en retrouve la trace à partir de thèmes aussi variés que abri, Ausweis, Boche, BOF, chanson, couvre-feu, école primaire ou marché noir, queue, rutabaga, ticket. Eric Alary traite évidemment les exodes, la ligne de démarcation, la gendarmerie nationale et Mme Vergez-Chaignon est particulièrement qualifiée pour rédiger les notices sur l'hôtel du Parc et sur Vichy, sur les vichysto-résistants, sur les cours de justice et l'épuration.

On s'arrêtera ici sur ce qui concerne la persécution et l'extermination des Juifs. L’une des questions soulevées dans la première partie par Bénédicte Vergez-Chaignon est celle de la complicité éventuelle de la France dans la « Solution finale ». Elle découle de la constatation que la très grande majorité des Juifs qui furent déportés de France vers les camps d'extermination ont été arrêtés et gardés par la police ou la gendarmerie françaises, qui se prêtèrent à cette tâche le plus souvent comme s'ils exécutaient n'importe quel travail. La police allemande ne disposait ni des effectifs, ni de la connaissance du pays et des gens nécessaires pour identifier et arrêter seule les Juifs. L'historienne souligne l'autonomie de décision du régime de Vichy qui, dès 
octobre 1940, entend exclure les Juifs français de l'État et de la société et favoriser la ré-émigration des Juifs étrangers. Le deuxième Statut en juin 1941, qui lui résulte de demandes allemandes, est décidé par Darlan et son gouvernement pour faire preuve de sa volonté sincère de collaboration au moment où la suprématie nazie apparait pérenne en Europe, mais aussi pour ne pas se laisser distancer en matière d'antisémitisme par l'occupant. Le recensement, les spoliations et les mesures d'internement préparent les premières déportations de 1942 en attendant que l'accord Oberg-Bousquet ne confie l'arrestation des Juifs étrangers et de leurs enfants en zone occupée et en zone libre aux forces de l'ordre de l'État français pour les envoyer, selon une note de Laval, dans un prétendu État juif que les Allemands voulaient constituer à l'Est de l'Europe. Par la suite, les protestations de certaines autorités religieuses et la désapprobation de l'opinion publique en zone libre conduisirent le gouvernement à limiter, mais non à supprimer, le concours de ses administrations et de sa police à de nouvelles arrestations. Mais, à partir de janvier 1944, la mainmise de la Milice sur le pouvoir répressif renforça les possibilités de déportation et de massacre, qui connurent un nouveau pic dans les mois qui précédèrent la Libération.

L'historienne insiste sur la responsabilité de l'appareil d'État français dans la déportation de 76000 Juifs vivant en France (dont 19500 citoyens français, 14000 enfants), à laquelle il faut ajouter 3000 Juifs morts dans les camps d'internement français et un millier de personnes exécutées ou massacrées en France par les Allemands ou leurs auxiliaires français. Le développement d'une violente campagne antisémite favorisée par Pétain et Laval dès l'été 1940 empêcha l'élan spontané de solidarité qui aurait pu se faire jour à ce moment, comme il se produisit à l'été 1942. Le fait même que les mesures antijuives aient été édictées par le Maréchal et son gouvernement contribua à abuser les Juifs eux-mêmes et à les inciter à adopter, contre leur intérêt vital, une attitude légaliste en se déclarant dans les commissariats ou les mairies.

Si les trois quarts des Juifs de France ont échappé à l'extermination, ce n'est pas aux gouvernements successifs de Vichy qu'ils le doivent, mais à létendue du pays qui permit à beaucoup d'entre eux de se cacher efficacement avec l'aide active de nombreux Justes, alimentée par la désapprobation massive de l'opinion après les rafles de 1942.

Ces quelques pages constituent un abrégé très efficace de la part de la question juive dans l'histoire du régime de Vichy, mais la suite de l'ouvrage y revient à plusieurs reprises. Mme Vergez-Chaignon avait souligné dans l'exposé précédent quaà l'époque de l'épuration, les policiers et gendarmes les plus compromis dans la traque aux Juifs ne furent punis que s'ils avaient fait preuve d'un zèle outrancier ou tiré profit personnellement de leur activité antijuive. Dans la seconde partie, elle rappelle la place centrale acquise au début des années 1980 par la mémoire de la Shoah, les procédures judiciaires pour crimes contre l'humanité, la reconnaissance par le président Chirac de la responsabilité de l'État français dans la déportation des Juifs, la création d'une mission sur la spoliation des biens juifs et celle de la Fondation pour la mémoire de la Shoah.

Enfin, le dictionnaire comprend un grand nombre de mises au point qui permettent au lecteur d'approfondir ses connaissances sur de nombreux points. 


\title{
LIBRAIRIE
}

Signalons les principaux articles concernant la persécution et l'extermination : aryanisation, Auschwitz, camps d'internement, Commissariat général aux questions juives, dénaturalisation, Drancy, étoile jaune, affaire du fichier juif, Justes, affaire Papon, sauvetages, Solution finale, statut des Juifs, Union générale des Israélites de France, Vel d'Hiv. D’autres notices apportent des données supplémentaires: attentats, Barbie, Bousquet, catholiques, délation, déportation, gendarmerie, Gestapo, otages, Pétain, polices, protestants, rafles, SNCF, Tsiganes.

Au total un travail très utile, complété par une bibliographie substantielle et un index.

\section{Dictionnaire de la violence}

\author{
Michela Marzano (dir.), Paris, PUF, 2011, 1546 p.
}

Par Frediano Sessi, Université de Mantoue

Presque quatre cents mots répertoriés, deux cents collaborateurs, ainsi qu'une grande quantité de croisements possibles permettant d'approfondir les fiches, caractérisent ce Dictionnaire, issu d'un projet interdisciplinaire, avec des rubriques dédiées à des penseurs (philosophes, anthropologues, psychanalystes, écrivains, etc.) qui ont élaboré des conceptions spécifiques de la violence ; d'autres rubriques qui développent des concepts tels que la barbarie, la fureur, la haine, la mort, l'animalité, etc. ; et d'autres encore, constituant de véritables essais rédigés par des spécialistes de secteurs particuliers, qui ont passé des années à étudier leur domaine ; enfin, des rubriques concernant des artistes tels que Picasso, Goya, Artaud, etc. ; le tout enrichi d'une vaste bibliographie où ont été sélectionnés les ouvrages de recherche les plus importants au niveau international sur les thèmes traités, ainsi qu'une série de renvois pour approfondir les idées dans le cadre du dictionnaire.

Pour lire et comprendre la violence, phénomène complexe, mais vieux comme le monde, de nouvelles questions se présentent à nous, maintenant que le problème de la violence est posé de façon entièrement nouvelle et inédite dans nos sociétés démocratiques, et que notre attention aux formes de vulnérabilité a augmenté dans le monde entier, tout comme s'est accrue la prise de conscience relative aux droits de l'homme.

Peut-on nourrir l'espoir, préconisé par les philosophes du Siècle des Lumières, d'éradiquer du monde toute violence, ou bien doit-on se résigner définitivement à la présence d'une ambivalence intrinsèque à l'être humain, qui ne serait donc jamais tout à fait bon ni tout à fait mauvais? Vu que de nos jours, tout comme au $\mathrm{XX}^{\mathrm{e}}$ 
siècle, la violence destructrice s'est déchaînée en partant de contextes extrêmement civilisés et cultivés, à l'intérieur desquels les êtres humains semblent faire partie de la communauté dans le respect des lois et de la raison au nom de valeurs universelles largement partagées, la question qui se pose n’investit pas seulement les individus, mais aussi les peuples et les nations de l'Occident comme de l'Orient, pour ne citer qu'un des contrastes culturels qui est d'une extrême actualité ; et cela concerne également les religions ainsi que la façon dont on les considère au sein de la vie quotidienne, dans les migrations et dans les confrontations ou dans les conflits entre collectivités et individus.

Le Dictionnaire a pour but principal d'essayer de fournir au lecteur des instruments de compréhension au sein de parcours disciplinaires et de langages différents, qui gardent néanmoins un lien étroit et une synergie. Comme le souligne la directrice de l'ouvrage dans son introduction, parler de violence veut dire entre autres s'interroger sur les frontières qui existent entre le moi et les autres, ainsi que sur l'ambiguïté de toute existence. Ces questions morales, existentielles et sociales prévalent toutefois sur les aspects liés à l'histoire, la sociologie et l'anthropologie. Le Dictionnaire attribue plus de poids et d'attention à la philosophie de la violence, à l'éthique et à la psychologie/ psychanalyse. Les rubriques historiques et anthropologiques sont rares et ont souvent un espace limité. Ceci n'empêche pas le Dictionnaire de la violence de se révéler un instrument de travail utile, qui ne peut manquer dans une bonne bibliothèque. Le recours final à l'index des noms et des thèmes ainsi que des concepts, tout comme les biographies des auteurs des fiches, sont importants, car ils nous permettent d'approfondir les thèmes traités en recourant aux ouvrages monographiques des auteurs en question.

Traduit de l'italien par Benedicte Cavanna

\section{Le Livre de Joseph}

\section{Bernard Dan, La Tour d'Aigues, L'Aube (Coll. «Regards d'ici »), 2011, 208 p.}

Par Albert Mingelgrün, Président de la Fondation de la Mémoire Contemporaine

Le titre retenu pour ce roman dissimule en fait le vrai héros-narrateur de l'histoire c'est-à-dire le dentiste parisien Jean-Paul Rakover, venu à Varsovie participer à un congrès scientifique et qui se voit, en raison d'une grève surprise, bloqué au moment du retour à l'aéroport de la ville qu'il ne pourra donc quitter qu'après un certain temps. Et c'est ici que le titre de l'ouvrage commence à donner signification au personnage 


\title{
LIBRAIRIE
}

de Rakover. Celui-ci va mettre en effet à profit son attente forcée pour entreprendre via son ordinateur portable et grâce aux liens électroniques, une véritable quête d'identité, retrouvant son grand-oncle Yosl Rakover alias Joseph Rakover, lequel a combattu les nazis dans le ghetto de Varsovie avant d'y mourir et laissé précisément le livre intitulé Yosl parle à Dieu. À partir de là et au fil d'extraits cités du livre en question, Jean-Paul va se retrouver lui-même imaginer son propre livre de bord : La vie et les étranges aventures de Jean-Paul Rakover de Paris, dentiste ; reconnaître les membres de sa famille en dehors de sa mère et se représenter en Israélien, bref se reconstituer en quelque sorte avant de reprendre l'avion pour Paris, à l'issue de la grève. Ce sera dès lors au cours de ce vol que s'accomplira l'ultime métamorphose et que surviendra l'ultime révélation. Métamorphose en effet puisqu'il affirme : « Mon cher Yosl, je sens ta main tendue vers moi : ta main tendue, c'est ce est - Rakover est Rakover, c'est-à-dire Yosl Rakover est Jean-Paul Rakover. Ou bien, Jean-Paul Rakover est Yosl Rakover. Notre contrat est scellé par ce petit verbe est »(p. 180-181); révélation également dans la mesure où « Yosl Rakover n'est qu'un personnage de fiction inventé de toutes pièces. Inventé par un écrivain qui s'est échappé» (p. 181). Et voilà comment un dentiste de stricte obédience professionnelle et au prénom papal est amené à s'inscrire dans la lignée des combattants du ghetto de Varsovie. L'humour et l'alacrité du style contribuent de leur côté à valider cette épopée identitaire.

\section{The Sign of the Cross. From Golgotha to Genocide}

Daniel Rancour-Laferriere, Piscataway, Transaction Publishers, 2011, 299 p.

\section{Jésus de Nazareth. Tome 2 : De l'entrée à Jérusalem à la Résurrection}

\author{
Joseph Ratzinger - Benoît XVI, Monaco, Le Rocher - Groupe Parole et Silence, 2011, 448 p.
}

Par Frans C.Lemaire

La Croix est devenue le symbole millénaire qui associe à la crucifixion de Jésus de Nazareth une double dimension, d'abord celle d'une mort volontaire apportant la rédemption des péchés et même la vie éternelle à chaque homme qui reconnaît en Jésus le fils de Dieu ${ }^{1}$, ensuite celle de cette même mort comme voulue par les Juifs

(1) « Dieu a tant aimé le monde qu'il a donné son Fils unique : ainsi tout homme qui croit en lui [...] obtiendra 
au prix d'une malédiction qui scellera définitivement le sort du « peuple déicide $»^{2}$. Il faudra deux mille ans et des millions de morts pour que l'ambiguïté de ce double symbole soit enfin dénoncée. Mais l'est-elle véritablement ? Tel est l'objet d'un livre particulièrement impressionnant, car son parcours « du Golgotha au Génocide » est soutenu par plus de mille notes renvoyant à une bibliographie de près de 800 titres.

Lauteur de ce travail a fait carrière comme professeur de langue russe à l'Université de Californie de Davis, particulièrement réputée dans le domaine de la recherche scientifique. Il a publié plusieurs ouvrages sur la culture russe, des Icônes à Tolstoï, en mettant particulièrement l'accent sur la relation avec le christianisme. Accédant à l'éméritat, il a entrepris ce travail gigantesque non tant par le nombre de pages (217 pour le texte proprement dit) que par l'importance, déjà soulignée, de la documentation qui le supporte. Ce livre s’adresse donc à la fois au spécialiste intéressé par des sources aussi nombreuses et pas toujours connues et à la fois au lecteur qui sattachera davantage aux grandes lignes de l'argumentation.

Trois mots reviennent sans cesse dans la première partie, presque au risque de lasser: masochism, paranoia et proxy. En rappelant la Crucifixion, la Croix est le symbole de la doctrine de repentir et de Rédemption qui en a été tirée par Paul de Tarse et les auteurs des évangiles, les Pères de l'Église et toute la tradition ensuite. Se plaçant en dehors des travaux sur le Jésus historique, le professeur Rancour-Laferriere se concentre sur la double éthique issue d'une théologie de la Croix alimentant à la fois les remords masochistes du chrétien et la malédiction paranoïaque des Juifs.

Après un premier chapitre consacré à une typologie des représentations de la Croix, l'auteur développe cette interprétation psychanalytique qui voit dans la Crucifixion, « un grandiose masochisme moral» (chapitre 2), annoncé et voulu par un Jésus ordonnant ainsi lui-même ses humiliations, ses souffrances et sa mort dans une éthique de non-résistance. Inspiré par ce modèle, le chrétien exemplaire, lecteur de L'Imitation de Jésus-Christ, doit porter la croix de ses repentirs, de ses renoncements, bref de sa soumission, mais la doctrine adoucit ce masochisme en célébrant, en particulier dans la liturgie et dans son discours théologique, la prise en charge par Jésus des péchés des chrétiens qui deviennent ainsi (chapitre 3) des Masochists by Proxy (masochistes par procuration).

Le Chapitre 4 est plus particulièrement consacré à la Résurrection comme Victoire de la Croix, tandis que le Chapitre 5 s’arrête sur les Croisades, dérive majeure

la vie éternelle » $(\mathrm{Jn}, 3,16)$, cité par Benoît XVI au début de sa première Lettre encyclique Dieu est amour, p. 5 (Salvator/Fidélité, 2006).

(2) Le même évangile de Jean l'annonce vingt versets plus loin $(J n, 3,36)$ : «Qui refuse le Fils ne verra pas la vie, la colère de Dieu demeure sur lui », ce que confirmera le célèbre verset de Matthieu $(27,25)$ : «Que son sang retombe sur nous et nos enfants », mais de cela la Lettre encyclique Dieu est amour de Benoit XVI ne fait aucune mention. 


\section{LIBRAIRIE}

transformant la Croix en épée (chapitre 5) malgré toutes les contradictions que cela entraîne vis-à-vis d'une doctrine d'amour du prochain, ennemis y compris.

Les trois derniers chapitres se penchent sur les conséquences de cette nondoctrine qui, en faisant des Juifs les ennemis perpétuels du christianisme, a justifié toutes les représailles. Le chapitre 6, intitulé Paranoia versus Paranoia by Proxy: The Cross and Christian Antisemitism, passe en revue les étapes de cette condamnation à perpétuité, en particulier telle qu'elle est exprimée dans les écrits accusateurs qui vont de Méliton de Sardes et Tertulien jusqu’à Drumont et Mein Kampf en passant par d'innombrables Adversus ou Contra Judaeos. Laccomplissement final auquel ils ont mené a sa propre croix que le chapitre 7 (The Holocaust: The Hooked Cross and Christian Antisemitism) analyse à travers les relations du christianisme avec le nazisme. Du côté protestant, les Deutsche Christen participent à l'aryanisation des écrits et de la liturgie en créant un Institut à cette fin, mais du côté catholique certains excès ne sont pas moindres, déclarant que Hitler est « le Sauveur, le Père et le Messie de la terre » et que la lutte contre le judaïsme fait du peuple allemand « le peuple élu de la promesse du Nouveau Testament. » Dix ans plus tard, le monde débarrassé définitivement de Hitler n'a pas, pour autant, tiré toutes les leçons de deux millénaires d'erreurs liées à la doctrine de la Croix (chapitre 8 : Unresolved Aftermath of the Holocaust) ${ }^{3}$.

Le supersessionisme (théologie revendiquant la substitution définitive du judaïsme par une «nouvelle » Alliance, un «nouveau » Testament) se trouve au cœur de ce drame. Même si cette théologie de la substitution semble remise en question, voire abandonnée par certaines déclarations ${ }^{4}$, elle est toujours présente et particulièrement à Auschwitz-Birkenau en s'efforçant de substituer une image victimiste chrétienne à la réalité historique d'un lieu où ce ne sont pas les victimes qui étaient chrétiennes, mais bien l'immense majorité des bourreaux ${ }^{5}$. On sait comment Jean-Paul II a multiplié les efforts en ce sens, brandissant une croix de 8 mètres de haut comme un signe de victoire là même où le christianisme a connu la plus grande défaite de son histoire. Si la manière de Benoit XVI est différente, elle n'en est pas moins ambiguë, révélant, au-delà de paroles de circonstances, très peu d'empathie et beaucoup d'incompréhension vis-à-vis des sensibilités juives. Vouloir à la fois béatifier Pie XII et écrire le texte d'une nouvelle prière pour que Dieu éclaire les Juifs afin qu'ils abandonnent le judaïsme ${ }^{6}$, témoigne de la permanence du refus de déraciner véritablement l'antijudaïsme chrétien.

(3) Il s'en faut de beaucoup puisque celui qui voyait en Hitler le Sauveur, le Père et le Messie en 1934 (Deutsche Sendung dans Werkblätter von Neudeutschland Älterenbund, n6, 1933/1934] n’est autre que Rudolf Graber, devenu après la guerre évêque de Regensburg et proche de Joseph Ratzinger qui en fera le concélébrant de la cérémonie l'intronisant archevêque de Munich en 1977.

(4) Nostra Aetate en 1965, Jean-Paul II à Mayence en 1980.

(5) Pas un seul d'entre eux n'a été exclu de la communauté chrétienne par une excommunication, mais beaucoup ont été aidés à fuir la justice.

(6) „Lasst uns beten auch für die Juden, dass Gott, unser Herr, ihre Herzen erleuchte, damit sie Jesus Christus als den Heiland aller Menschen erkennen“" (Benoit XVI, 6 février 2008) 
Pour l'auteur de The Sign of the Cross, les textes évangéliques qui racontent le drame de la Croix et leur interprétation antijudaïque sont à la base d'une « paranoïa par procuration » accompagnant « le masochisme par procuration » de la doctrine rédemptrice. Dans les dernières lignes de sa conclusion (p. 259), Daniel RancourLaferriere ne cache pas son scepticisme vis-à-vis des tentatives de rapprochement œcuménique, en particulier judéo-chrétien, aussi longtemps que ne sera pas ouvertement posé le problème des textes évangéliques eux-mêmes et de leur contenu accusateur.

Après avoir fait durant dix-neuf siècles du Nouveau Testament une parole de Dieu et de Dieu seul et ainsi, de la foi, la source de toute vérité, le christianisme romain se trouve confronté au renversement de ses postulats. La vérité est devenue plus importante que la foi ou en tout cas, le mépris de la vérité si souvent pratiqué au nom de la foi n'est plus accepté. La résistance reste forte cependant, surtout lorsqu'il s'agit de la responsabilité fondamentale liée aux textes évangéliques et à ce que la « tradition » a fait durant près de deux mille ans d'un antisémitisme qui aurait pu rester une simple rivalité sectaire anecdotique. «De larges sections du Nouveau Testament devraient être censurées ou, à tout le moins, réécrites. Mais après cela, restera-t-il encore des «Écritures » ? », demande Rancour-Laferrière en posant ainsi la question ultime de leur banalisation parmi les milliers d'autres textes « sacrés » issus de l'imagination humaine.

Il est intéressant de rapprocher ces réflexions conclusives de la constatation faite par Christian Stückl, régisseur depuis 1990 de la Passion d'Oberammergau. Lorsqu'il accède à ce poste grâce à la confiance que lui accorde une municipalité qui avait été dominée jusqu'alors par d'anciens nazis non repentis, opposés à tout changement, Stückl fait de l'élimination de l'antisémitisme du spectacle un de ses premiers objectifs. Il arrive très vite à la conclusion que le problème n'est pas tant dans le livret de l'ancien curé d'Oberammergau, Alois Daisenberg, utilisé depuis 1850, que dans les textes évangéliques eux-mêmes, en particulier le fameux verset 27, 25 de Matthieu: "Que son sang retombe sur nous et nos enfants. » Informée de cette orientation, la Conférence des évêques allemands envoie à Oberammergau un proche ${ }^{7}$ du cardinal Ratzinger qui s'oppose à tout changement dans ce sens et ce n'est finalement qu'en 2010, 55 ans après la Shoah, qu'un spectacle pleinement débarrassé de ses stéréotypes antisémites, y compris évangéliques, a pu être réalisé.

Du côté protestant, une initiative remarquable a eu lieu à Dresde en l'an 2000 avec la présentation d'un nouvel oratorio de la Passion ${ }^{8}$ dont le livret établi par le

(7) Il s'agit de l'exégète Rudolf Pesch qui a interrompu en 1984 une brillante carrière universitaire pour travailler à plein temps pour le cardinal Ratzinger à travers le mouvement des Katholische Integrierte Gemeinde qu'il soutient depuis 1978 et l'Institut de théologie opposé aux facultés universitaires récemment créé à la Villa Cavaletti de Castel Gandolfo.

(8) Mathias Drude, Für deine Ehre habe ich gekämpft, gelitten. Stationen der Passion Jesu (2000) pour soprano, baryton, récitant, chœur et orchestre. 


\section{LIBRAIRIE}

théologien Hartwig Drude, oncle du compositeur, intègre les résultats d'une étude sur le procès de Jésus, récemment publiée en allemand". Pour la première fois, ce ne sont plus les Juifs qui sont responsables de la crucifixion et la mort de Jésus. Après un choral et un prologue dans le style luthérien, le récitant avertit le public du changement apporté au récit de la Passion :

À la base de cette musique de la Passion se trouvent des récits et des prières de la Bible. Ils ne suivent cependant pas, sans réserve, les rédactions des évangélistes. Ces récits ne font pas que rapporter ce qui s'est passé, ils veulent aussi expliquer. Face à la mort de Jésus, ils veulent disculper le pouvoir romain. C'est pourquoi ils présentent Pilate (Pilate Poncius, préfet romain de Judée de 26 à 37 è.c) comme un simple exécutant, un homme hésitant, voire un philosophe raffiné. Il était, en réalité, tout à fait différent. Malgré cela, les narrateurs chrétiens font paraître, contre toute vérité ou vraisemblance, « les Juifs » et même « le peuple juif tout entier » comme les forces déterminantes de la mort de Jésus.

«Contre toute vérité et toute vraisemblance.» Nous voilà loin des évangiles dont Dieu serait le seul auteur. L'exégèse biblique catholique s'est dans sa grande majorité ralliée ces dernières décennies ${ }^{10}$ à cette lecture historique-critique, entamée deux siècles plus tôt dans les milieux intellectuels luthériens et, selon laquelle les textes du Nouveau Testament sont humains et rien qu'humains, pour le meilleur et pour le pire, ce pire dont ce nouveau livre entreprend l'analyse et la psychanalyse.

Est-ce pour autant l'heure de vérité pour le Vatican? Si la publication d'un Jésus de Nazareth en deux volumes" ${ }^{11}$ ar Benoit XVI est, ainsi qu'il le souligne lui-même, l'œuvre personnelle d'un cardinal et non celle d'un pape, il n'en reste pas moins que les convictions qu'il affirme, largement opposées à l'exégèse historique-critique, ne peuvent qu'être celles du magistère actuel dénonçant régulièrement le «relativisme » et le «pluralisme ». Comment dans ce contexte, le cardinal Ratzinger résout-il le problème du conflit opposant les Juifs à Jésus, tel qu'il est raconté par les auteurs des évangiles. Bien que la volonté des Juifs de tuer Jésus apparaisse dès le verset 5 , 18 dans l'évangile de Jean, cet aspect est ignoré et ce n'est quà la page 213 qu'est posée pour la première fois la question : «Qui étaient les accusateurs, qui a insisté pour que Jésus soit condamné à mort ? » Passant en revue les réponses que l'on trouve dans chacun des évangiles, l'auteur affirme que, « contrairement à ce que le lecteur moderne serait

(9) Haim Cohn [juriste israélien d'origine allemande (1911-2002)], The Trial and Death of Jesus, New York, Harper \& Row, 1967 ; Chaim Cohn, Der Prozess und Tod Jesu aus jüdischer Sicht, Berlin, Suhrkamp, 1997.

(10) A Marginal Jew, Rethinking the Historical Jesus, tel est le titre d'un monumental ouvrage en quatre volumes (New Haven, Anchor Yale Bible Series / Reference Library, 1991-2009), d'un exégète catholique américain, John P. Meier, disponible aujourd'hui en français sous le titre Un certain Juif, Jésus (Paris, Le Cerf, 2004-2009). L'autre auteur important est Raymond E. Brown, publié en français chez Bayard.

(11) Tome 1, Du baptême dans le Jourdain à la Transfiguration, Paris, Flammarion, 2007.

Tome 2, De l'entrée à Jérusalem à la Résurrection, Monaco, Le Rocher, 2011. 
tenté d'interpréter », l'expression « les Juifs » que l'on rencontre un grand nombre de fois chez Jean, «n'indique en aucune manière le peuple d'Israël comme tel [...], mais a une signification précise et rigoureusement limitée : il désigne par-là l'aristocratie du Temple. » On ne peut affirmer davantage le contraire de lévidence puisque le texte de Jean fait clairement la distinction entre « les Juifs » mentionnés 61 fois et les prêtres et scribes du Temple spécifiés séparément 21 fois, sans parler des Pharisiens désignés 19 fois. Dès le tout premier verset qui parle des Juifs (1, 19), Jean désigne séparément les prêtres et les lévites.

Le problème de la foule qui réclame la mort de Jésus devant Pilate est transformé de la même façon en un « groupe de partisans de Barabbas qui se sont mobilisés pour l'amnistie [...], mais pas le peuple juif comme tel » (p. 214) alors qu'en réalité Matthieu dit bien explicitement en 27,25 « tout le peuple » et lui fait revendiquer toute la responsabilité de son crime: «que son sang soit sur nous et nos enfants.» Pour écarter un sens aussi évident, le cardinal Ratzinger affirme (p. 215) que contrairement à Jean et Marc, « Matthieu n'exprime pas un fait historique, et quant au sang, il ne s'agit ni de vengeance, ni de punition, mais de réconciliation [...], c'est en fonction de la foi qu'il faut lire la parole de Matthieu sur le sang. Ce n'est pas une malédiction, mais une rédemption, un salut » (p. 216).

Que durant dix-neuf siècles, l'Église n'ait pas lu ces paroles en fonction de la foi, mais en fonction de la haine issue de sa doctrine, se trouve ici totalement passé sous silence. C'est, une fois de plus, l'image du christianisme tel qu'il devrait être et non pas tel qu'il a été.

Nous nous trouvons donc en présence d'un type de pensée pour laquelle l'exaltation de ce qui n'appartient pas à l'histoire - la Résurrection, l'Ascension, la parousie, la venue du Royaume de Dieu et du salut des hommes - s'accompagne du silence sur ce qui s'est effectivement produit, voire de sa négation ${ }^{12}$, une attitude que l'on retrouvera pleinement dans la volonté de béatifier un Pie XII présenté comme tel qu'il aurait dû être pour mieux effacer ce qu'il a été.

(12) C'est ce que réalise notamment l'auteur aux ordres, Rudolf Pesch, écrivant en 1990 un texte niant l'antisémitisme dans la Passion d'Oberammergau, tout en défendant le maintien du verset de la malédiction (Mt, 27, 25) et publiant en 2005 un livre encore plus radical intitulé Antisemitismus in der Bibel ? Das Johannesevangelium auf dem Prüfstand (Augsburg, Sankt Ulrich, 2005) devenu en français, sans point d'interrogation : L'évangile n'est pas antisémite, Saint Jean soumis à l'examen (Paris, Desclée de Brouwer, 2007). 


\title{
Tiempo Pasado, Cultura de la memoria y giro subjetivo - una discusión ${ }^{1}$
}

\author{
Beatriz Sarlo, Buenos Aires, Siglo XXI editores, 2007, 168 p.
}

Par Mylène Herry, Université de Toulouse - Le Mirail

«C'est plus important de comprendre que de se souvenir même si, pour comprendre, il faut aussi se souvenir ${ }^{2}$.»

Beatriz Sarlo

Directrice de la revue culturelle et politique Punto de vista $a^{3}$ pendant trente ans (1978-2008) et ancienne professeure de littérature à l'Université de Buenos Aires, Beatriz Sarlo incarne aujourd'hui, à travers ses nombreux essais, l'intelligentsia critique en Argentine. Dans cet ouvrage de 2007, divisé en six parties dont je respecterai l'ordre, elle étudie la persistance des actions du passé sur le présent à travers la mémoire individuelle et collective et reconsidère le binôme Histoire/mémoire. Ainsi, dans le contexte post-dictatorial de l'Amérique latine, explore-t-elle comment la reconstruction démocratique passe par une récupération discursive, définie, en partie, par le genre testimonial, et multiplie-t-elle les références théoriques et littéraires afin de confronter des réalités multiples.

\section{TIEMPO PASADO 4}

Beatriz Sarlo pose ici la problématique de la tension systématique des champs mémoriels, du conflit entre ceux qui veulent oublier, et ceux qui maintiennent le souvenir et proposent la/une parole. Le souvenir, même s'il n'est pas toujours convoqué ni verbalisé, se fait présent et incontrôlable. Alors, la mémoire est un devoir postérieur où le témoignage, comme source privilégiée et unique de certaines expériences, permet aujourd'hui la condamnation du terrorisme d'État. La restauration de la raison et la confiance du sujet ne doivent pas engendrer pour autant, selon l'auteur, une industrie de la mémoire nourrie par une société de plus en plus voyeuriste.

\section{CRITICA DEL TESTIMONIO : SUJETO Y EXPERIENCIA ${ }^{5}$}

Le combat pour l'Histoire est donc un combat identitaire où le sujet convertit

(1) Temps Passé, Culture de la mémoire et orientation subjective - une discussion.

(2) Citation de Beatriz Sarlo (p. 26) qui introduit la trame de son livre et avoue l'étroite correspondance avec les convictions de l'écrivaine américaine Susan Sontag.

(3) J'indique, par souci informatif, l'adresse web de la revue citée : http://www.revistapuntodevista.com.mx/ portal/index.php.

(4) Temps passé.

(5) Critique du témoignage : sujet et expérience. 
l'expérience personnelle, viscérale, en expérience commune du dire. Le langage ne peut pas reconstituer l'intensité des faits du passé, mais inscrit l'expérience dans la temporalité du souvenir. La référence au choc, traité, entre autres, par Walter Benjamin, permet à Beatriz Sarlo de considérer que la narration, comme mise en voix, engendre la séparation du corps, et, donc, éloigne de l'expérience. Le sujet témoin relate sa vérité en fonction, d'une part, de ce qu'il a vu, entendu et/ou vécu, et, d'autre part, du temps de latence écoulé depuis lors. Il peut ainsi représenter, mais non remplacer, celui qui a vécu dans sa chair l'expérience. Par exemple, et comme le rappelle l'Argentine, Primo Levi se considère victime de la Shoah, mais rejette le caractère exhaustif de son témoignage, ayant échappé, lui, à la mort. Il ne peut, dans cette optique, donner aux lecteurs que « la matière première de l'indignation ${ }^{6}$. » Il n'y a pas de représentation vicaire du condamné ; seuls les rescapés sont en mesure de témoigner à la place des morts. Ainsi, la prosopopée, comme modèle testimonial, tend à privilégier la dimension éthique et collective du discours et relève, selon les références à Paul de Man, d'un artifice rhétorique. Pour Beatriz Sarlo, le témoignage ne peut pas être à l'origine du discours historique jugeant qu'on ne peut alléger la vérité de l'expérience du sujet, et encore moins sa souffrance. Enfin, et pour contrer la position de Paul Ricœur dans Temps et récit ${ }^{7}$, le témoignage doit examiner et non négliger cette subjectivité discursive depuis laquelle l'imagination naît et constitue une arme sociale pour les laissés pour compte.

\section{LA RETORICA TESTIMONIAL ${ }^{8}$}

Beatriz Sarlo part de la signification du verbe se souvenir, considéré en Amérique latine, comme un motif de restauration des liens sociaux et communautaires, détruits par la violence gouvernementale et par l'exil consécutif. Les témoignages dans ce sens permettent d'exhiber, du point de vue des subalternes, les crimes des dictatures dont ils ont été victimes. Alors, le présent de l'énonciation marque une rhétorique de la persuasion où le présent dirige le passé, dont la distorsion permet une cohérence discursive plus évidente. Le récit complet favorise, sur le modèle romantico-réaliste, l'inclinaison du discours pour les détails malgré leur caractère argumentatif et peu significatif. Le passé devient alors illusoire et loin du caractère premier de l'expérience. Cependant, la mémoire anachronique révèle les événements et porte une responsabilité morale collective dont le savoir sur la répression, par exemple, est irremplaçable. Cette mémoire a de l'intérêt dans le présent au même titre que l'Histoire ou l'art. Selon Beatriz Sarlo, le discours testimonial, parfois proche des « limbes interprétatifs » (p. 94), doit permettre non seulement d'accéder à la condamnation des coupables des violences commises, mais aussi, et surtout, de donner aux récepteurs de la matière pour comprendre les victimes.

\footnotetext{
(6) Citation de Primo Levi, utilisée comme sous-titre de la 3ème section de la seconde partie de l'ouvrage.

(7) Paul Ricoeur, Temps et récit I, Paris, Seuil, 1991.

(8) La rhétorique testimoniale.
} 


\section{LIBRAIRIE}

\section{EXPERIENCIA Y ARGUMENTACION'}

Lauteure traite le témoignage, sous d'autres perspectives, à travers deux textes: La bemba d'Emilio de Ípola (1978) et Poder y desaparición; los campos de concentración en Argentina de Pilar Calveiro (1998). Ces écrits littéraires proposent de privilégier la portée intellectuelle et analytique sur l'expérience du sujet témoin. Alors, la construction testimoniale n'est plus seulement narrative ; elle doit produire des connaissances. Ainsi, la première œuvre citée pose-t-elle la théorie de la rumeur en milieu carcéral et la seconde mêle-t-elle les résultats d'une recherche doctorale à l'expérience personnelle des camps.

\section{POSMEMORIA, RECONSTRUCCIONES ${ }^{10}$}

Ensuite, et tout en examinant les positions des professeurs James Young et Marianne Hirsch, Beatriz Sarlo s'intéresse à la post-mémoire dont le caractère identitaire, affectif et moral, se transmet de génération en génération. Alors, le souvenir public ou familial de faits tragiques ou heureux, transmis par les institutions ou les ascendants, marque la mémoire du sujet. Lauteure reprend ici l'exemple de Mauss d'Art Spiegelman. En effet, cet ouvrage de littérature graphique mêle la reconstruction de l'histoire orale familiale, où la subjectivité est impliquée, et la problématique de l'historien posée ici à travers le contexte de la Shoah. Mais, cette mémoire « de seconde génération » (p. 128) est fragmentée et irrésolvable si l'on considère le système de décalages et de ponts idéologiques, discursifs et sociaux, vacillant entre les époques, les souvenirs, les traumatismes et l'oubli.

\section{MAS ALLA DE LA EXPERIENCIA ${ }^{11}$}

La dernière partie peut être considérée comme une réflexion conclusive sur le rôle de transmission de la littérature, menée par un narrateur souvent étranger à l'expérience, et dont l'autorité, en tant que « maître d'ouvrage », permet de prendre possession des réalités afin de les transcender.

(9) Expérience et argumentation.

(10) Post-mémoire, reconstructions.

(11) Au-delà de l'expérience. 


\title{
Le Piège des loups. \\ Les 175 maisons de la Gestapo en France
}

\author{
Dominique Sigaud, Paris, Stock, 2012, 378 p.
}

Par Corinne Benestroff, Université Paris 8

Dominique Sigaud dresse l'inventaire des 175 maisons de la Gestapo en France et ainsi que l'écrit Patrick Modiano : « on se dit qu'au moins les lieux gardent une légère empreinte des personnes qui les ont habités ${ }^{1}$.» S'il s'agit bien d'un relevé systématique de ces maisons, le lecteur, qu'il soit chercheur ou non, familier de la période ou pas, y trouvera bien plus que cela.

Ily a d'abord la somme informative. Lauteur retrace dans son chapitre introductif l'histoire de la Gestapo, la police secrète d'État créée par Hermann Goering en 1933. La fusion de différents services aboutira à la création de la Sipo-SD (Sicherheitspolizei : police de sécurité, Sicherheitsdienst : service de sécurité.) Les Français utiliseront le terme générique de Gestapo pour désigner les différentes forces d'occupation et de répression. Cette première partie donne une vision d'ensemble de l'appareil de répression, militaire, administratif et policier, qui forme un maillage très dense sur tout le territoire. L'ouvrage est ensuite organisé en trois grandes sections chronologiques à l'intérieur desquelles sont décrits les 22 régions et 175 postes et antennes du $S D$ Gestapo et Grenzpolizei, la police des frontières.

Mais, cette enquête est bien plus qu'un simple relevé. L'hommage aux résistants, cette armée des ombres inattendue, est le fil rouge autour duquel s'organisent les descriptions : « Je sais », écrit Sigaud, « il me semble essentiel que ce soit bien clair, ce que nous devons aux uns et aux autres, à leur courage. » (p. 37) La diversité de cette armée est très bien restituée. S'y côtoient ceux dont l'Histoire a retenu les noms et les autres : ouvriers, cheminots, enseignants, étudiants, agriculteurs, femmes au foyer, fonctionnaires luttant sur tous les fronts. Leurs actions sont multiples diffusion de tracts, sabotages, fabrication de faux papiers, aide aux réfractaires, renseignement, sauvetage des Juifs -, au sein de structures plus ou moins organisées. De la résistance civile à la lutte armée, des actions isolées aux maquis, on suit l'évolution et la construction de ce qui s'appellera la Résistance.

Ponctué d'extraits d'archives, de circulaires administratives, de propos tenus par les prisonniers et les tortionnaires, le texte tresse en un savant mélange les dire des uns et des autres, retrace les destinées souvent tragiques et restitue la complexité de cette

(1) Patrick Modiano, Dora Bruder, Paris, Gallimard, 1997, p. 30. 


\section{LIBRAIRIE}

période obscure, l'intrication des liens entre la machine de répression de l'occupant et l'administration française, la cohabitation dans une même aire géographique des délateurs et des résistants. Lauteur n'adopte pas une vision surplombante et donne à voir par bribes la densité de ce temps particulier, le régime de la terreur installé à demeure, l'effroi sous la tranquillité des apparences. En effet, au cœur de cet inventaire méthodique, règnent la douleur et l'effroi qui se sont abattus sur tous ceux qui furent traqués et persécutés : opposants, résistants, Juifs. La torture est aussi omniprésente et l'on ne peut s'empêcher de penser aux voisins de ces maisons qui entendaient les cris des suppliciés. Mais, l'ouvrage questionne aussi notre rapport à la mémoire de ce temps-là, notre faculté et notre désir d'oubli. Dans la plupart des cas, ces maisons cossues ont retrouvé leur vocation première de bâtiments publics ou habitations particulières sans que rien ne vienne indiquer ce qui s'y passa, comme si cet effacement s'inscrivait dans l'ordre naturel des choses. Sans grandiloquence, ni manichéisme, cette recherche minutieuse et sensible des traces nous offre un objet mélancolique. Les photographies des maisons, des résistants, des victimes de la persécution y contribuent, convoquant les disparus. Celles des SS sont là également comme pour nous rappeler que ces maisons, si familières, furent aussi les antichambres des camps. Derrière elles, se profilent donc l'univers concentrationnaire et celui de l'extermination. En les arrachant à l'oubli, dans une démarche poétique, l'auteur de Franz Stangl et moi ${ }^{2}$ écrit comme au seuil de l'absence la post-mémoire au présent et nous dit : « Passant, souviens toi !»

\section{Le Mythe du grand silence. Auschwitz, les Français, la mémoire}

\section{François Azouvi, Paris, Fayard, 2012, 477 p.}

Par Paul Bernard Nouraud, École des Hautes Études en Sciences sociales de Paris

Si le ton et le titre du Mythe du grand silence sont polémiques, c'est que, selon son auteur, on mésestime rétrospectivement la qualité de la réception d'Auschwitz dans la France de l'immédiat après-guerre. Or, en l'espèce, François Azouvi juge qu'il y a matière à révision. De ce sentiment est issue une recherche de grande ampleur, dont l'élaboration a produit une écriture claire et précise, à la fois inquiète et sûre, qui fait retentir chaque texte qu'il présente, et tous ensemble. Ces textes, ce sont les articles,

(2) Dominique Sigaud, Franz Stangl et moi, Paris, Stock, 2011. 
les éditoriaux et les critiques qui dessinent les contours de la réception d'Auschwitz en France ; textes qui tentent de mettre à jour par la parole l'événement même qui a d'un seul coup provoqué et révoqué la parole - qui l'a suffoquée, comme l'écrivait Sarah Kofman'. On pourrait dire en somme que Le Mythe du grand silence s'emploie à « débiaiser » notre perspective du passé. Ce n'est pas parce qu'on n'en parlait pas, c'est parce qu'on n'en parlait pas comme on en parle maintenant que le mythe du grand silence français sur la destruction des Juifs d'Europe a pu s'énoncer et recevoir peu à peu un à-valoir devenu aujourd'hui doxa.

«Je voudrais montrer que c'est une "légende" » (p. 13), écrit Azouvi, parce que, « qu'on le veuille ou non, le processus par lequel l'extermination des Juifs est entrée dans l'espace français est normal. » (p. 14) Cette «normalité » du processus pose d'autres problèmes, mais c'est bien en première instance cette réalité historique, culturelle et sociale qu'il fallait rétablir, réalité de la prise en charge du caractère inédit d'Auschwitz par les intellectuels dans un premier temps, par l'opinion ensuite, et cela, dès son lendemain.

Aussi François Azouvi entreprend-il l'analyse des basculements du processus de mémorisation du génocide que la France d'après 1945 a connus à travers la réception d'un certain nombre d'œuvres marquantes de cette période; basculements qui auraient selon lui eu des effets différents, voire atténués, si les Français n'avaient été disposés à basculer avec eux. Cette analyse des discours de la réception tend simultanément vers une pensée de la disponibilité imaginative saisie par l'inimaginable. Ce livre constitue ainsi une généalogie de l'appréhension valant peu à peu conceptualisation et, au sens fort, au sens où Paul Ricœur pense la dette, prise en compte; travail de honte et de culpabilité, dit Azouvi.

Le Mythe du grand silence rapporte comment, historiquement, culturellement et politiquement, cette conscience a été préparée jusqu’à la rendre, pour citer un autre mot cher à l'auteur, mature. Or si ces basculements résonnent, c'est, écrit Charlotte Delbo exprimant, aux dires mêmes d'Azouvi, le cœur de sa thèse, «parce qu'il y a boîte de résonance », et cette boîte, ajoutait Delbo, «c'est la conscience.» (p. 325) Par ces mots, l'auteur d'Auschwitz et après rendait alors compte du formidable impact de la diffusion en France au début des années 1980 de la série télévisuelle américaine Holocauste, non sans s'inquiéter des conséquences qu'une telle appropriation de l'événement allait avoir sur sa perception.

Mais c'est que tout basculement, rappelle Azouvi, même s'il résonne plus justement qu'Holocauste, comporte des risques de mésinterprétation, puis de mésusage du génocide, que les dispositions du public soient favorables ou défavorables. Il faut citer ici quelques-uns de ces événements qui tirent leur qualité d’avoir «brusqué

(1) Sarah Kofman, Paroles suffoquées, Paris, Galilée, 1986. 


\section{LIBRAIRIE}

le moment », pour paraphraser Focillon. François Azouvi note ainsi qu'avec Nuit et Brouillard (1956) d'Alain Resnais, un vocabulaire du sacré apparaît pour la première fois massivement dans le discours critique ; avec la pièce d'Hochhuth, Le Vicaire, en 1963, qui dépasse en importance, selon Azouvi, le procès Eichmann de 1961 puisqu'elle divise cette fois la presse chrétienne, c'est le thème de la concurrence des victimes et bientôt de leur mémoire qui se fait jour² ${ }^{2}$ Treblinka, de Jean-François Steiner prépare la rhétorique de la levée des tabous et brouille la réception, la même année 1966, d'Eichmann à Jérusalem d'Hannah Arendt, sur la question de la « collaboration » juive ; avec Shoah (1985) de Lanzmann, « quelque chose aussi s'achève », écrit Azouvi, « parce que le génocide a trouvé son œuvre. » (p. 362), et avec elle l'accomplissement de son unicité, de sa sacralité ; avec Déportation et génocide, qu'Annette Wieviorka publie en 1992, qui confère enfin au mythe du grand silence une caution historique, indispensable à son intégration dans l'espace public.

En portant attention à ces temps forts de la mémoire d'Auschwitz, François Azouvi prend soin de ne pas céder pour autant à une périodisation plaquée sur le cours historique ${ }^{3}$. C'est qu'en effet, rappelle-t-il, telle qu'elle est admise aujourd'hui, cette périodisation puise davantage au registre de la psychanalyse qu'à celui de l'interprétation historique. À travers son schéma ternaire (traumatisme, refoulé, retour du refoulé) s'est imposée sur les études historiques «la puissance du paradigme psychanalytique dans l'interprétation du nazisme » (p. 301), et ceci dès la fin des années 1960, lorsque les premières traductions des essais de Bruno Bettelheim ont paru en France 4 .

Reste que parallèlement aux productions artistiques, aux recensions médiatiques, à la pensée historique et philosophique faisant état de la maturation de la mémorisation d'Auschwitz en France, on constate le retard (on serait tenté de dire ici l'immaturité) de la puissance publique sur la question. Si l'auteur a raison de souligner que «s'il y a en France un “syndrome de Vichy”, il n’y a pas de “syndrome de la Shoah”. » (p. 284),

(2) À ce propos, l'auteur passe peut-être un peu vite sur cette notion qui recouvre à partir de la fin des années 1990 un phénomène complexe appelant une étude socio-historique plus approfondie quant à la légitimité des attentes suscitées. Le fait de prendre exemple du livre d'Olivier Pétré-Grenouilleau sur les traites négrières (Les Traites négrières. Essai d'histoire globale [2006], Paris, Gallimard, coll. « Folio Histoire », 2009), et du procès auquel il a donné lieu, illustre cette indétermination. Sans doute Pétré-Grenouillau, au plan statistique, « établissait[-il] la réalité » (p. 398), comme l'indique Azouvi, mais c'est tout de même un tour de force que de consacrer plus de 600 pages à un objet historique tel que le fait esclavagiste sans - à aucun moment s'interroger sur la signification de celui-ci, sinon sur un mode approximatif.

(3) Philippe Mesnard perçoit mêmement le danger d'obscurcissement qu'une périodisation hâtive fait porter sur l'intelligence de l'événement. Cf. Philippe Mesnard, « Approches critiques à propos de trois notions fortes des études sur le témoignage », in Luba Jurgenson, Alexandre Prstojevic, Des Témoins aux héritiers. L'Écriture de la Shoah et la culture européenne, Paris, Pétra, (Coll. « Usages de la mémoire »), 2012, p. 51-84.

(4) Lesquelles paraissent aujourd'hui non seulement inefficientes, mais souvent franchement déplaisantes pour ne pas dire infamantes pour les victimes. Cf. le chapitre vi du Cœur conscient (Le Cœur conscient. Comment garder son autonomie et parvenir à l'accomplissement de soi dans une civilisation de masse [1960], trad. Laure Casseau, Paris, Robert Laffont, 1978, en particulier p. 278 sqq.) ou son avant-propos au témoignage de Miklós Nyiszli (Auschwitz: A Doctor's Eyewitness Account [1946], Greenwich, Fawcett Crest, 1960, p. I-XIV). 
il apparaît de plus en plus nettement que le premier corrèle logiquement le second. L'exemple du discours du président Giscard d'Estaing à Auschwitz-Birkenau en 1975, bien qu'injustement oublié, ne peut éclipser la nomination de Maurice Papon, par le même président, au ministère du Budget trois ans plus tard, au sein du gouvernement de Raymond Barre.

Le fait qu'il y a dix ans encore on enseignait fortement dans les Instituts d'Études politiques que Vichy avait été une parenthèse, anticonstitutionnelle de surcroît (la Constitution violée au moment des pleins pouvoirs, l'honneur était sauf), ne peut être compris qu'articulé à la montée en puissance du «travail de la honte». La permanence, jusque dans les années 1970, de hauts fonctionnaires coupables de près ou de loin de ce qui avait désormais un nom et une reconnaissance, a indubitablement entretenu l'impression d'un grand silence; d'un syndrome l'autre en somme. Et s'il est vrai qu'au lendemain de la guerre, les hommes de l'État n'ont pas été muets sur le génocide, cet empressement n’a plus eu sous la v ${ }^{\mathrm{e}}$ République la même allure, et ce, jusqu’au discours du président Chirac, qui constitue, aux yeux d'Azouvi, la concrétisation de la maturation qu'on a dite, la reconnaissance qu'une opinion désormais attendait.

Mais il y a plus que cette distinction des deux syndromes entre lesquels pourtant la disjonction fut ténue. Il s'agit de la réaction des intellectuels catholiques dans l'immédiat après-guerre qui occupe l'auteur dans la première partie de son ouvrage. S'y rencontrent les trois éléments qui sous-tendent tout l'essai et avec lui l'histoire de l'intégration du génocide à ses représentations : la puissance et les limites de l'imagination humaine, la fonction appréhensive de l'eschatologie chrétienne, la réinscription du génocide dans le registre du sacré.

« Ce sont avant tout les limites de l'imagination humaine qui ont étouffé une connaissance qu'il n'était pas possible de se procurer» (p. 22), écrit Azouvi. Par parenthèse, il faudrait dire ici que ce sont ces mêmes limites qui rendent les hommes perméables à ce mythe, en particulier les jeunes générations. La prise de conscience de cette histoire, et parfois de cette mémoire, par un adolescent (prise de conscience que l'école en l'effectuant ne passe pas de fait sous silence) peut prendre à ses yeux une telle forme de scandale et de sidération qu'il se trouve tout disposé à croire qu'on les lui a cachées. Et c'est qu'en effet, un temps, celui de son enfance, on les lui a cachées ; on a délibérément soustrait à son imagination l'inimaginable, conscient que celui-ci ne pouvait être intégré qu’après maturation et constitué qu’après qu'il ait été inscrit, pour parler une nouvelle fois avec Ricœur, dans un récit, celui-ci fût-il historique ou fictionnel.

C'est du reste ces termes ricœuriens qu'emploie Azouvi pour décrire les premières phases du processus d'appréhension d'Auschwitz lorsqu'il écrit qu'« amorcé au lendemain de la guerre, ce processus de mise en fiction va être dans les années cinquante le principal mode d'entrée de l'événement dans la conscience française. » (p. 120) 


\section{LIBRAIRIE}

Il est d'ailleurs intéressant de noter que, du côté de la conscience américaine, le critique d'art et ami d'Arendt Harold Rosenberg perçoit, dans Commentary, le procès Eichmann alors en cours comme une opportunité de « mettre en récit » le génocide, le procès « prenant en charge la fonction de la poésie tragique ${ }^{5}$. " Coïncidant avec Azouvi, il constatait qu'il « est déjà difficile de se rappeler combien peu l'on savait avant le Procès de ce qui avait été fait aux Juifs d'Europe. Non pas que les faits de la persécution n'étaient pas disponibles ", mais davantage que " ce corps croissant de connaissances ${ }^{6}{ }$ n'avait pas encore pénétré les consciences, en particulier les consciences juives américaines des lecteurs de Commentary. «Sans doute aucun crime n’a été si bien documenté et si vaguement appréhendé ${ }^{~ », ~ e ́ c r i v a i t ~ R o s e n b e r g . ~ A u s s i ~}$ toute la valeur du procès tenait-elle en ceci qu'elle permettait enfin une narration ou, pour reprendre ses mots, une tragédie, dans laquelle les témoins allaient jouer le rôle du Chœur antique et la présence physique d'Eichmann réinscrire ses crimes dans l'histoire.

Un besoin d'imaginer et de se représenter Auschwitz, on le sait, parcourt ainsi toute l'histoire de sa mémorisation en Occident. Ce que l'on savait moins avant l'ouvrage d'Azouvi, c'est que cette injonction sétait faite jour dans l'opinion dès l'ouverture des camps, et que les penseurs catholiques avaient trouvé moyen d'y répondre. « Il y a une raison à la précocité avec laquelle les intellectuels chrétiens se sont emparés du génocide ", écrit Azouvi, « et en ont compris immédiatement la spécificité: Alain Besançon l'a parfaitement dit, c'est qu'ils disposent d'un "schéma théologique cohérent de la Shoah”, celle du Serviteur souffrant du Second Isaïe. » (p. 53) Ce n’est pas une mince affaire, insiste l'auteur, que la spécificité de la destruction des Juifs d'Europe ait été reconnue si tôt, et de surcroît parmi les non-Juifs. Au point que cette reconnaissance trouve son prolongement historique et, comme dans le cas de l'État français, sa concrétisation, dans l'abandon, par le concile Vatican II, de la rhétorique antijuive de l’Église.

Cependant, les imaginations catholiques de Maritain, de Claudel, de Madaule et de Gabriel Marcel font preuve d'élasticité autant que de rigidité : l'inscription du génocide dans un schéma théologique ne contredit-elle pas la qualité «spécifique» de son appréhension? Concevoir de la sorte la « reprise » du génocide dans l'eschatologie chrétienne n'est peut-être guère charitable à l'endroit de penseurs qui ont sincèrement œuvré dans le sens d'une Église dépouillée de ses ambiguïtés et pour tout dire, renouvelée, conscients que cet effort de l'Église pèserait aussi sur l'opinion française. Reste qu'il y a quelque chose de saisissant dans la sûreté avec laquelle ces mêmes penseurs ont révisé leurs cadres imaginatifs et conceptuels : ils les ont recadrés, mais

(5) Harold Rosenberg, « The Trial and Eichmann », Commentary, vol. 32, n 5, novembre 1961, p. 369-381, ici p. 369.

(6) Ibid., p. 374.

(7) Ibid. 
ne les ont pas fait sauter. La contrition même, la repentance encore s'inscrivent jusque dans le rituel de cette théologie. Comment se fait-il qu'ils aient été ébranlés ${ }^{8}$, certes oui, mais qu'ils n'aient pas douté, radicalement douté ? Auschwitz a ébranlé le monde, mais pas la foi chrétienne; pas Rome, pas Mauriac. On attendait d'eux un sacrilège ; ce fut un sacrifice qu'ils rendirent; autre moyen de produire du sacré, rappelait Bataille.

Car c'est bien de sacré qu'il s'agit. C'est d'ailleurs en commentant les propos tenus par le Père Dupuy en 1984, deux ans avant qu'il ne prononce à Auschwitz-Birkenau la déclaration de repentance de l'Église, que François Azouvi prend acte qu' « une sorte de théologie négative est ici en germe, qui n'est pas un moindre danger que sa transformation en un "holocauste" inscrit dans l'économie divine. » (p. 343) Ce lien d'un absolu à l'autre indique une parentèle qui était jusqu'alors restée inaperçue et une telle coïncidence mérite d'être davantage étudiée. N'est-il pas frappant de voir qu’à quarante ans de distance Jacques Maritain et George Steiner recourent à un même schéma explicatif du génocide et à des conclusions similaires quant à son « usage » et aux « usages de sa mémoire ${ }^{9}$ »?

Dans cette tendance à l'absolutisation du fait humain en ce qu'il a de « déshumain », pour parler avec Fédida, il semble y avoir une aspiration à faire d'Auschwitz non pas plus, mais autre chose que ce qu'il n'est, de le soustraire à la fois à l'investigation historique et à l'imagination fictionnelle. Il semble y avoir, en toute fin de compte, une volonté de faire quelque chose d'Auschwitz. Ce qui achoppe et achoppera toujours, pourtant, dans la fiction, dans les essais et à l'école, c'est que oui, il faut faire quelque chose d'Auschwitz, car sa connaissance est nécessaire, son travail impératif, et qu'en même temps celle-ci n'est rien d'autre qu'« une connaissance inutile ${ }^{10}$ ».

(8) Il était important de rappeler en l'occurrence l'acuité de pensée de l'un de ces penseurs, Max Picard, dont Le Visage humain ([1929] Paris, Buchet/Chastel, 1962) a notamment influencé Emmanuel Levinas. Mais il est aussi intéressant d’observer comment, à la fin de cette puissante analyse du fait nazi qu'est L'Homme du néant ([1946] Neuchâtel, La Baconnière, 1963), celle-ci se trouve en quelque sorte désarmée devant le « remède » au nazisme qu'elle se sent obligée de proposer. Le caractère sommaire de la compréhension chrétienne peut alors se résumer en une phrase : à l'exemple du mal, il faut désormais proposer celui du bien, le phénomène d'adhésion inconditionné à l'exemple étant identique.

(9) «Ce n'est pas, écrit Maritain dans une lettre à Pierre Visseur, secrétaire de la conférence de Seelisberg, le 27 juillet 1947, parce que [les Juifs] ont tué le Christ, c'est parce qu'ils ont donné le Christ au monde que la rage de l'antisémitisme hitlérien a traîné les Juifs sur toutes les routes de l'Europe dans les ordures et le sang. » (cité p. 82) Lettre à laquelle font écho ces lignes de Steiner : « It is not, I believe, as deicide, as "God killer", that the Jew has been loathed and feared in the Christian civilization of the West lalthough that hideous attribution does play its part). It is as inventor of God; it is as spokesman for and remembrance of an almighty, all-seeing, all-demanding Deity. » George Steiner, «The Long Life of Metaphor: An Approach to the "Shoah" », in Berel Lang (dir.), Writing and the Holocaust, New York/Londres, Holmes \& Meier, 1988, p. 154-171, ici p. 164.

(10) Charlotte Delbo, Auschwitz et après. Tome II : Une Connaissance inutile [1970], Paris, Minuit, 2004. 


\section{Triangle rose}

\section{Michel Dufranne, Bruxelles, Quadrants, 2011, 146 p.}

Par Anthony Michel, CREM (Metz) - IPSE (Luxembourg)

La bande dessinée est un média que l'on aurait pu croire uniquement destiné aux histoires humoristiques ou épiques. Michel Dufranne, lui, s'en est inspiré pour raconter une histoire vraie, celle de la discrimination des homosexuels via le « Paragraphe 175 » du Code pénal allemand, qui en a envoyé plusieurs milliers en déportation.

Dès la première de couverture, les notions de discrimination et de déportation sont visibles puisque l'illustration présente l'homosexuel à la fois habillé dans le tristement célèbre pyjama rayé et sur lequel est cousu un triangle rose (signe distinctif au même titre que l'étoile jaune pour la population juive). De plus, l'exclusion est aussi de mise au sein même de la population allemande des années 1930-1940, puisqu'aucune personne autour de lui (une dizaine) ne le regarde.

Pour traiter ce sujet, l'auteur a décidé de raconter l'histoire d'un lycéen qui, pour un devoir, interroge son grand-père, Andreas, Berlinois, homosexuel et rescapé des camps. Les souvenirs liés à son histoire vont alors refaire surface. Un simple prétexte pour rappeler le sort des homosexuels dans les sociétés allemande et française jusqu'aux années 2000.

L'histoire se déroule à notre époque puisque dès les premières pages, on remarque le terme «wiki » (p. 3), relatif aux nouvelles technologies de l'information et de la communication, mais aussi à la distance et au désintérêt entre l'actualité et l'histoire que l'on peut comprendre grâce à l'expression prononcée par un personnage adolescent : « J'en ai rien à foutre de ses camps. » (p. 3) Par la suite, on apprend que ni le jeune Alexandre ni son père ne connaissent le passé d'Andreas qui n’a jamais voulu parler de son expérience carcérale, ni du motif de sa déportation. Dès l'explication de la démarche de son petit-fils, l'aïeul, au visage très marqué, se durcit dans ses propos, en reprenant par exemple : «On dit Seconde Guerre mondiale quand on est optimiste ! » (p. 10) ou encore lorsqu'il refuse d'être enregistré pour l'interview. Cela montre un refoulement de son passé et une volonté de ne laisser aucune trace des événements qu'il a vécus.

Lincompréhension est instantanée lorsqu'Alexandre apprend que son grand-père a été enfermé dans les camps alors qu'il était Allemand. Sur la joue de ce dernier, une larme apparaît... C'est le premier chapitre « Les années brunes », début des souvenirs du rescapé. 
Dans les années 1930, Andreas est un dessinateur de publicités ; il en a même réalisé certaines pour le parti d'Hitler. On apprend immédiatement qu'il est homosexuel, à la fois par une vignette sur laquelle il embrasse un autre homme et dans une bulle où il est écrit : «Alors, maman a enfin laissé sortir son gros pédé de fils? » (p. 17)

Nous nous trouvons alors au cour de la communauté homosexuelle de Berlin, de leurs sujets de discussion, de leurs lieux de rencontre, des propos tenus à leur égard et de leur langage, assez vulgaire. La discrimination des Juifs est présente dans leurs discours, mais divise plusieurs membres de leur groupe. Andreas fait partie de ceux qui trouvent cela justifié.

La mère d'Andreas est au courant de l'orientation sexuelle de son fils et a peur qu'il ne soit découvert. En guise de réconfort, il explique qu'il ne « connait pas plus homosexuels que Röhm et ses petites sections d'assaut (SA). » (p. 58) Le titre du chapitre fait référence aux « chemises brunes », surnom donné aux miliciens chargés de faire office de service d'ordre dans les meetings et d'agresser les adversaires des nazis dans la rue. En juin 1934, « les nazis montrent leur vrai visage [...] ils ont traqué les SA et arrêté Röhm sous prétexte qu'il est homosexuel. » (p. 71)

Hitler au pouvoir, les dénonciations apparaissent. La concierge de l'immeuble d'Andreas le dénonce à la police. Il est donc convoqué, mis en garde et par la même occasion se fait licencier. Le climat sociopolitique n'est plus du tout favorable à la communauté homosexuelle.

Malgré la tentative de faire croire à son hétérosexualité, en se promenant avec une femme, il est tout de même arrêté et mis en prison. Jugé, mais relaxé, il sera peu de temps après de nouveau arrêté par la Gestapo et envoyé dans un camp de concentration. C’est le début du deuxième chapitre : « Les années noires ».

Les pyjamas rayés, le triangle rose, les visages creusés, les corps squelettiques et les barbelés : voilà le paysage du camp de concentration dans lequel a vécu Andreas à cause de son homosexualité, pendant que d'autres étaient torturés (souvent jusqu’à la mort) au siège berlinois de la Gestapo. Les déportés sont traités d'« anormaux » et de « musulmans » (p. 108), surnom donné aux individus sur le point de connaître la mort. On apprend alors le sort qui leur est réservé dans le camp de Neuengamme : pas de couverture dans les baraquements de peur qu'ils ne fassent des « saloperies en dessous » (p. 109) ou encore, ils doivent marcher trente kilomètres pour assouplir les chaussures des soldats, en ayant des « chaussures trop petites et deux pieds droits. » (p. 113) Les années passent et se ressemblent : les conditions de vie épouvantables, le travail, la torture. Le 19 avril 1945, les survivants sont évacués par des bateaux-prisons coulés par les Alliés. « La poignée de rescapés » (p. 118) a enfin gagné sa liberté.

Le troisième chapitre « Les années de larmes », évoque le retour à la vie civile d'Andreas et la reconstruction du pays. Un service se met en place pour indemniser les victimes de la déportation, mais évoquer ce traumatisme dont il sort à peine est déjà 


\title{
LIBRAIRIE
}

difficile pour lui. Quand on en vient au motif de l'incarcération, le « Paragraphe 175 », cela ne lui permet pas d'être reconnu comme une vraie victime, car il était considéré comme « criminel relevant du droit commun. » (p. 126) Il est encore stigmatisé dans les rues à son retour, lui est rentré vivant et non pas les « vrais Allemands qui ont crevé comme des rats. » (p. 134)

Andreas explique que le 22 mai 1949, à la veille de la naissance de la République fédérale allemande, le « Paragraphe 175 » ne sera pas abrogé ; il risque d'être considéré comme récidiviste en encourant une lourde sanction. Son départ d’Allemagne est inéluctable.

Au cours d'une commémoration française en 1985, la mémoire des déportés homosexuels n'est toujours pas reconnue et entre en conflit avec celle de « ceux qui ont réellement souffert de la guerre. » (p. 138) Son pyjama rayé rangé au placard, Andreas avoue « la victoire des nazis qui les ont tous assassinés dans les camps. » (p. 139) Pour le rescapé, les déportés homosexuels sont les grands oubliés de l'histoire.

Dans son discours du 24 avril 2005, Jacques Chirac reconnait la déportation des homosexuels, bien trop tard pour Andreas. Cette annonce précède le dernier chapitre « Devoir de mémoire - Droit à l'oubli », qui nous ramène à la diégèse initiale, celle de l'interview par le petit-fils Alexandre. Le vieil homme ne répondra pas aux questions et lui dira que tout est dans les livres...

Le contexte dans lequel s'inscrit la diégèse (les années 2000), le choix du support plutôt inattendu (la bande dessinée) et le fait d'intégrer narrativement la communauté homosexuelle (on partage leur vie, leurs discours et leur langage) font de cet ouvrage un excellent vecteur actuel pour traiter d'un sujet «passé sous silence» (à la fois par les témoins et par les pouvoirs politiques), voire polémique il y a encore quelques années seulement. Ainsi, cette contribution participe au devoir de mémoire d'une catégorie de victimes liée au nazisme, faiblement reconnue et médiatisée jusquà aujourd'hui.

\section{Qui écrira notre histoire? Les Archives secrètes du ghetto de Varsovie}

\author{
Samuel D. Kassow, Paris, Grasset, 2011, 593 p.
}

\section{Par Judith Lindenberg, EHESS}

Louvrage de Samuel Kassow, intitulé Qui écrira notre histoire ? et sous-titré Les Archives secrètes du ghetto de Varsovie est un livre en forme de poupées russes, dans lequel chaque sujet est contenu dans un autre plus vaste. Pour raconter l'entreprise d’un historien, Emmanuel Ringelblum, et de son collectif Oyneg Shabes (« Joie du 
Shabbat »), l'ouvrage plonge ses racines dans une histoire culturelle bien plus large, celle de l'intelligentsia juive polonaise à l'origine, dans la première moitié du vingtième siècle, de l'Histoire juive moderne. Pendant la guerre, le petit groupe réuni sous le nom d'Oyneg Shabes a recueilli et produit des archives sur les conditions de vie des Juifs en Pologne et sur le ghetto de Varsovie pendant toute la durée d'existence de celui-ci, de 1940 à 1943. Cette chose incroyable - la production d'archives dans des conditions de vie telles que celles du ghetto et le fait que celles-ci nous soient parvenues - ne peut en effet être appréhendée sans en retracer les origines.

Partant de Simon Doubnov qui, le premier, insista à la fin du siècle précédent, en Russie, sur la nécessité de la collecte (zamling) de matériaux bruts pour écrire une histoire qui ne soit pas religieuse, mais sociale, cette exigence fut ensuite portée par Meir Balaban et Isaac Shiper, considérés comme les fondateurs de l'histoire juive polonaise.

À l'instar de Doubnov, leur innovation fut de s'appuyer sur des sources non religieuses, mais ethnographiques. Ringelblum, comme Balaban, Shiper et de nombreux intellectuels juifs polonais de la première moitié du siècle, était originaire de Galicie ; en raison de la situation politique particulière de cette région, intégrée à l'Empire austro-hongrois jusquà 1918, les Juifs tout en étant imprégnés de folklore yiddish, y jouissaient d'un accès à la culture et à l'éducation privilégié par rapport au reste de la Pologne, qui leur permettait d'acquérir des instruments critiques à même de penser la situation des Juifs polonais dans leur ensemble. Pour tous ces historiens, une telle conception était profondément liée à une volonté politique de prise en main de la destinée du peuple juif : faire l'histoire de ce peuple était ressenti comme une mission nationale, car elle permettrait une conscience et une affirmation de l'identité juive. Mais cette identité, tout en revendiquant son autonomie, se pensait comme inextricablement liée à celle des Polonais. Ringelblum n'eut de cesse de tenir ce lien, jusquà son dernier ouvrage emblématiquement consacré aux relations judéopolonaises ${ }^{1}$.

Face au courant assimilationniste incarné par le Bund, et «à l'instar de Doubnov, Ringelblum pensait que la conscience historique pouvait offrir un rempart culturel aux Juifs laïques qui rejetaient à la fois la religion et l'assimilation ${ }^{2}$. » Le courant politique dans lequel s'inscrivait Ringelblum, et auquel il fut toujours fidèle, le Poaley Tsion des sionistes de gauche, cherchait à tenir ensemble des positions apparemment contradictoires : la place des Juifs en Pologne et l'aspiration à un territoire national. Mais au fond, comme le montre Kassow, ces contradictions tenaient à ce que «le sionisme galicien se souciait moins d'émigration immédiate en Palestine que de nouvelles définitions de la judéités.»

(1) Emmanuel Ringelblum, Polish-Jewish relations during the Second World War, Joseph Kermish et Shmuel Krakowski, Evanston, 1974.

(2) Samuel D. Kassow, Les Archives secrètes du ghetto de Varsovie, Paris, Grasset, 2011, p. 27.

(3) Ibid., p. 42. 
En dessinant le portrait intellectuel de Ringelblum, Kassow souligne le caractère exceptionnel de l'Histoire juive telle qu'elle émerge dans la première moitié du vingtième siècle et dont il est l'héritier. L'historien y revêt un rôle politique central : en faisant connaître aux Juifs leur histoire, il leur fait prendre conscience de leur existence en tant que groupe en dehors de la religion et leur confère un rôle dans l'histoire polonaise. Cette conception de l'Histoire est d'autant plus remarquable qu'elle surgit dans un contexte d'où elle était absente, la religion juive étant fondée sur une vision anhistorique, dans laquelle les événements sont pris dans une répétition. Lémergence d'une pensée historique, qui signe l'avènement d'une culture juive laïque et son entrée dans la modernité, s'intègre dans un courant intellectuel plus large, incarné notamment par le YIVO, fondé en 1925 à Vilnius et rassemblant différentes disciplines des sciences sociales fondées sur un souci commun de l'ethnographie. Enfin, le dernier élément de cette conception moderne de la culture juive est la place accordée au yiddish. Aussi bien au YIVO que dans les recherches de nombreux historiens se consacrant à l'histoire juive, le yiddish apparaît alors comme la pierre de touche de lajudéité polonaise, car elle concentre les dimensions populaires, culturelles et politiques. Cet intérêt pour le yiddish permet aussi de mesurer l'ambiguïté et la complexité de la culture juive polonaise, qui est loin d'être socialement unifiée. En effet, il s’agit de la langue des couches populaires tandis que les Juifs assimilés parlent généralement le polonais ; mais ce sont des intellectuels provenant de ces mêmes classes assimilées qui vont s'employer à promouvoir l'usage de la langue du peuple - et d'une vaste littérature depuis le XIX ${ }^{\mathrm{e}}$ siècle - comme ciment de l'identité juive laïque. Comme dans la collecte des sources, ce sont les masses juives, leurs usages et leur histoire qui sont mises au centre de l'histoire.

C'est dans ce contexte culturel que s'inscrit donc l'itinéraire de Ringelblum, chez qui l'on retrouve toutes ces influences. L'imbrication entre histoire et politique qui caractérise sa pensée se retrouve dans ses activités professionnelles : contrairement à ses prédécesseurs, il n’a jamais occupé de poste universitaire, mais il enseignal'histoire dans un lycée Borokhov, du nom du mentor de son groupe politique à l'origine du sionisme de gauche. Parallèlement, il débuta dans les années trente une carrière institutionnelle à la tête de plusieurs organismes de secours: en premier lieu l'Aleynhilf, qui jouera un rôle de premier plan dans le ghetto et dans l'organisation d'Oyneg Shabes. Il travailla aussi au Joint Distribution Committe ; cela lui conférera une grande expérience dans l'aide humanitaire, qui se révélera également fondamentale dans le ghetto. C'est finalement cette complémentarité entre la pensée et l'action qui permit à Ringelblum d'y accomplir son travail et de fonder Oyneg Shabes. Mais l'action précède : lors de la création de celui-ci, en octobre 1940, l'Aleynhilf devient d'emblée non seulement un moyen d'entraide, mais aussi une conscience morale, un contrepoids au Judenrat et à ses dérives.

Dès le début de la guerre, Ringelblum avait tenu un journal. Mais c'est en se fondant sur le travail de l'Aleynhilf qu'Oyneg Shabes se forma : par la distribution de l'aide, ses membres étaient directement en contact avec toutes les couches du ghetto, 
surtout les plus défavorisées. Dans les conditions de vie du ghetto, l'idée d'un travail collectif, qui dérivait directement de l'influence ethnographique du « collectez, ils feront le tri ${ }^{4} »$, s'imposait. Les membres d'Oyneg Shabes, dont beaucoup avaient collaboré au YIVO avant-guerre, furent recrutés peu à peu. En collectant le plus de « matériaux bruts » possible (tracts, affiches), en poussant les gens à écrire (journaux), en réalisant des entretiens, Oyneg Shabes mit sur pied une archive gigantesque grâce à laquelle la vie dans le ghetto nous est aujourd'hui connue. En racontant l'aventure d'Oyneg Shabes, Kassow restitue le quotidien du ghetto notamment à travers le prisme de la trajectoire de ses principaux membres, d'origines sociales et savantes diverses. Mais pas seulement : fidèle à sa conception d'une histoire « du peuple et par le peuple », Oyneg Shabes recueillit des témoignages de Juifs de toute la Pologne, et particulièrement des shtetlekh, afin de constituer un tableau le plus complet possible de la vie en temps de guerre.

Au fur et à mesure cependant de la guerre et de la mise en place de la Solution finale, la vision du collectif changea, et de là sa finalité :

Jusqu'au début de 1942, Oyneg Shabes, comme Peretz durant la Première Guerre mondiale, avait cru que la fin de la guerre verrait la renaissance de la communauté juive en Pologne, les archives servant à étayer la conscience nationale tout en étant un moyen de créer un « passé utilisable » pour l'avenir. Au printemps de 1942, cependant, les artisans d'Oyneg Shabes commencèrent à se dire qu'ils étaient peut-être, au contraire, en train d'écrire le dernier chapitre des huit siècles d'histoire des Juifs polonais ${ }^{5}$.

À partir de la vague des déportations massives, la constitution des archives semblait devenir dérisoire et entrer en concurrence avec une lutte constante et désespérée pour la survie. Mais c'est justement là que se révèle la foi des membres du collectif en leur mission. Larchive prenait un sens nouveau au moment où les Juifs polonais risquaient de disparaître : « même si les Juifs polonais ne survivaient pas, les archives restaient nécessaires. Sans cela, la postérité lirait les archives des tueurs, mais oublierait les voix des victimes ${ }^{6}$. » L'infime probabilité de réussite d'une entreprise dont chaque phase tenait à un fil - non seulement la collecte elle-même, mais l'enfouissement des archives en trois parties, et leur découverte successive grâce aux trois survivants, Rachel Auerbach, Hersch Wasser et sa femme Bluma ${ }^{7}$ - confère à l'ensemble un caractère miraculeux. En cela réside le tour de force vertigineux d'Oyneg Shabes, que Kassow donne à lire à la fois comme un épisode de l'histoire du génocide et comme le point d'orgue d'une réflexion sur l'histoire qui parcourt toute l'historiographie juive.

(4) Ibid., p. 32.

(5) P. 306.

(6) Ibid., p. 473.

(7) « Auerbach et Wasser persistèrent et, avec l'argent du Jewish Labor Committee de NY, les recherches commencèrent enfin pour de bon dans l'été 1946. [...] Dès avant la fin de la guerre, la Commission qui, outre Auerbach, comprenait des chercheurs comme Philip Friedman, Joseph Kermish et Nathan Blumenthal, recueillit des journaux, des Mémoires et des témoignages de survivants. » 


\title{
LIBRAIRIE
}

À travers ces archives, Ringelblum s'approcha au plus près de son rêve de l'avant-guerre d'une histoire juive pour et par le peuple. Ce sont ces circonstances exceptionnelles et dramatiques qui lui permirent de réaliser sa plus grande œuvre, tout en écrivant la dernière page de l'Histoire de laquelle elle était le produit.

En replaçant l'aventure d'Oyneg Shabes à la fois dans la trajectoire d'Emmanuel Ringelblum et à travers celle-ci dans le cadre historique plus large de l'histoire juive de la première moitié du vingtième siècle et des courants idéologiques qui la traversèrent, Kassow montre comment les archives secrètes du ghetto et le travail de Ringelblum furent l'aboutissement de plusieurs décennies de réflexions politiques et historiques sur la place des Juifs polonais. La coïncidence entre le moment de ce travail historique et de la disparition du groupe qui en est l'objet est troublante et invite d'autant plus fortement à lire cet ouvrage non seulement comme le récit, très bien écrit, d'un moment exceptionnel, mais surtout comme la restitution d'une leçon d'histoire qui reste à méditer.

\section{Une Éthique du regard}

\author{
Sylvie Rollet, [Coll. «Fictions pensantes »), Paris, Hermann, 2011, 276 p.
}

Door Fabian Van Samang, Historicus

In Une éthique du regard. Le cinéma face à la Catastrophe, d'Alain Resnais à Rithy Panh ('Een ethiek van de blik. De bioscoop oog in oog met de Catastrofe, van Alain Resnais tot Rithy Panh') analyseert de Franse filosofe Sylvie Rollet hoe massaal geweld met enige overtuigingskracht visueel kan worden overgedragen van de historische actoren op het hedendaagse publiek. Hoewel haar studie in feite over de filmische verbeelding van genocide gaat, laat ze dit begrip bewust achterwege en geeft ze de voorkeur aan de term 'Catastrofe' (met hoofdletter). 'Genocide’ weerspiegelt immers de biologische categorieën van de dader, beargumenteert Rollet, terwijl het begrip 'Catastrofe' verder gaat dan de historische (genocidale) gebeurtenissen - de term heeft betrekking op een meta-vernietiging, een evenement waarvan de implicaties voelbaar zijn over de grenzen van tijd en ruimte heen.

Centraal in het boek staat de aanname dat het beleefde geweld niet gere-presenteerd kan worden, omdat het nooit present, nooit aanwezig is geweest. Slachtoffers blijken immers vaak onmachtig - en daders vaak onwillig - om de beleefde ervaringen mee te delen. Precies daarom zal een strikt logisch-deductief of chronologisch verhaal niet volstaan om de 'Catastrofe' inzichtelijk(er) te maken. Een filmische verbeelding, 
waarbij de kijker wordt uitgedaagd, veelal door een pijnlijke confrontatie met leegtes, stiltes en verrassende momenten, kan echter wél tot reflectie aanzetten. Ze kan toegang bieden tot een ruimte waarin wordt geluisterd en geargumenteerd, door alle partijen - de menselijke interactie die zo wordt gerealiseerd is een politieke en ethische daad bij uitstek. 'Door een beroep te doen op onze mogelijkheid om vanuit het standpunt van "de ander" te denken en door van de vernietiging van de mens(elijk)heid een kwestie te maken van onze mens(elijk)heid, kan de cinema een "getuigende kunst" worden die tegemoet komt aan de wens van Kertész: om de Catastrofe in de cultuur te laten binnentreden' (p. 248-249).

Het mechanisme van transmissie illustreert Rollet aan de hand van zeven (documentaire) films die de jongste halve eeuw werden gerealiseerd door cineasten als Alain Resnais (Nuitet Brouillard, 1955), Harun Farocki (Images du mondeet inscription de la guerre, 1988; En sursis, 2007), Atom Egoyan (Calendar, 1994; Ararat, 2002), Claude Lanzmann (Shoah, 1985) en Rithy Panh (S 21, 2002). Hoewel elke film in een ander tijdsgewricht tot stand kwam, een verschillende uiting van massaal geweld tot onderwerp heeft en het evenement telkens vanuit een andere wetmatigheid aan het publiek voorlegt, is de selectie niet willekeurig. Elk werk visualiseert immers het onzegbare, stelt Rollet. Stuk voor stuk overbruggen ze de kloof tussen het feit en het evenement; en net daarom heeft elk besproken stuk een historische en ethische revolutie ontketend.

'Une éthique du regard' situeert zich op het raakvlak van historiografie en wijsbegeerte. Volgens Rollet menen geschiedkundigen in archivalische bronnen al te snel de onweerlegbare bewijzen voor verleden gebeurtenissen te kunnen ontdekken. En net daarom is het werk van Resnais en Farocki zo revelerend: ze vechten het mythologiserende bronnenfetisjisme aan en leggen de grenzen van de historische re/ constructie bloot - zij het door het visualiseren van wat verdwenen is, zij het door de leegte-, maar ook inhoudsscheppende montagetechnieken. Het ontmythologiseren van de bron ('anarchivisme') helpt in ieder geval de kijker uit zijn onverschilligheid weg te halen. In Egoyans oeuvre wordt de overdracht van de leegte, die door het massale geweld werd nagelaten, gevisualiseerd door blanco's (entre-images), die de verbeelding van de kijker moeten prikkelen. De schijnbaar chaotische opbouw van 'Calendar' bakent door de gedwongen speurtocht van het publiek naar de inhoud en betekenis van het verhaal de mogelijkheidsvoorwaarden van een gedeelde herinnering af. In 'Shoah' wordt de kloof met het ongrijpbare verleden gedicht door wat Rollet de 'dialectique de l'incarnation' noemt: door de niet-intentionele, plotse handelingen van de historische actoren - die worden versterkt door een landschap dat de Shoah uitademt - maakt de kijker zich een niet pre-existerende, maar door de montage vormgegeven waarheid eigen. Zo komt in de eenzaamheid en de angst die Simon Srebnik uitstraalt, wanneer hij door een schimpende groep antisemieten wordt omringd, de nauwe vervlechting van het anders-zijn en het gedood worden, 'toen' en 'nu', tot uiting. De getuigen in Shoah zijn geen getuigen an sich, maar brengen 'in opdracht' van zij die niet overleefden een boodschap over die wetenschappelijk niet 


\section{LIBRAIRIE}

kan worden be- of ge-grepen. Rithy Panh legt van zijn kant de wordingsgeschiedenis van de dader bloot; met 'S 21' (de naam verwijst naar één van de detentiecentra van de Rode Khmer) creëert hij een tegenherinnering, die de geschiedvervalsing van het Cambodjaanse regime moet tegengaan. Daarom laat hij dader en slachtoffer samen-denken, toont hij hoe het lijf (de houding, de stem, toon en intonatie) van de voormalige beul zijn oude ideologie weerspiegelt (het lichaam wordt zo een 'corpsarchive') en personaliseert hij het slachtoffer tot een individu waarmee de aangekeken kijker zich kan identificeren.

Rollets studie zet ongetwijfeld aan tot nadenken. Het boek bevat verrassend nieuwe invalshoeken, legt verbanden die verre van alledaags zijn en daagt uit om de besproken films met een kritische(re) bril te (her-)bekijken. Het bespeelt de grenzen van het zeg- en denkbare, van het feit en het evenement, van geschiedenis en geschiedschrijving. En het (her-)vormt de wijze waarop we genocides terugblikkend kunnen, mogen, moeten beoordelen.

Toch lijken enkele van Rollets basisassumpties de toets van de kritiek moeilijk te kunnen doorstaan. Dit komt ten dele door de onnauwkeurigheid waarmee ze haar concepten definieert. Wat moeten we ons concreet voorstellen bij de 'Catastrofe' of het 'evenement' waarover ze het heeft? Indien we het optimaal willen begrijpen, moeten we immers weten over welk fenomeen we precies nadenken. Is het de altijd subjectieve ervaring van de overlevenden/getuigen, die door hun ongewenste aanraking met een verleden realiteit een complexe en voor buitenstaanders altijd onkenbare relatie met dat verleden hebben opgebouwd (een 'transcendentale reductie', zoals Maurice Merleau-Ponty die begreep?). Maar indien we zouden kunnen intreden in de ervaringswereld van de overlevende/getuige, zouden we 'het evenement' daarmee ten volle hebben begrepen? Zouden we de wordingsgeschiedenis ervan hebben blootgelegd, de omvang, de gevolgen? Zouden we er het instrumentarium mee kunnen creëren, dat we nodig hebben om soortgelijke 'Catastrofes' in de toekomst te vermijden? Indien niet, dan dient een deel van 'het evenement' wellicht elders te worden gezocht. Is de 'Catastrofe' misschien het geheel van handelingen, zowel die op korte als op wat langere termijn, die leidden tot het genocidale fenomeen dat we vandaag - al was het maar door zijn enorme omvang - nog steeds niet kunnen begrijpen? Maakt de (onbegrijpelijke) afstandelijkheid van de omstaanders er deel van uit? De wijze waarop we er vandaag (niet) mee omgaan? Hoe het wordt gedacht, vergeten, aangevoeld of als symbool wordt gebruikt? Of is het de eindeloos veranderende interactie van dit alles? Rollet verduidelijkt dit niet en lijkt verschillende verklaringsfactoren toe te passen op een term die ze zélf voortdurend van gedaante laat veranderen. Daarom lijkt het alsof het onderwerp van reflectie aan onze kennis ontsnapt, niet omdat het an sich helemaal onkenbaar is, maar omdat het door de auteur permanent met andere inhouden wordt bekleed.

"De uitspraken van iemand mogen onoprecht zijn, maar in de stijl van zijn taal ligt zijn wezen open en bloot," schreef de Duitse taalfilosoof Victor Klemperer 
in zijn befaamde Lingua Tertii Imperii (Klemperer, LTI, 2000, p. 25). Een in het oog springend kenmerk van Rollets taalgebruik is de (schijnbare) contradictie. Haar boek rijgt uitdrukkingen aaneen die in hun conventionele betekenis nauwelijks kunnen worden samengedacht, maar in hun nieuwe context toegang lijken te verlenen tot nooit bewandelde betekenisvelden. Zo heeft ze het onder meer over de 'overdraagbaarheid van het onoverdraagbare' (une transmissibilité de l'intransmissible) (p. 94), de 'aanwezigheid van de afwezigheid' (cette présence de l'absence) (p. 154), zingeving in een zinloos universum ('car tout finit par prendre sens dans un univers où le sens échappe') (p. 173), de orde in het chaotische narratief (un ordre se dégage toutefois de cette narration chaotique) (p.158). Versterkt door het veelvuldige gebruik van aanhalingstekens (die suggereren dat de lezer op zoek moet gaan naar een - niet nader omschreven - achterliggende betekenis), ontstaat zo een semantische vervuiling, die aanzienlijk afwijkt van het dwingende wetenschappelijke imperatief dat de vorser tot maximale conceptuele duidelijkheid en verfijning aanzet.

Enkele stellingen zijn bovendien zo abstract en veralgemenend, dat ze onmogelijk nog kunnen worden weerlegd (of bevestigd). Waarom zou het beeld van een moeder en haar kind voor een kerk verwijzen naar de afwezigheid van de mannen die het kerkgebouw hebben opgetrokken, maar door de genocide zijn weggevaagd? Waarom zou hun ààn-wezigheid niet geïnterpreteerd kunnen worden als een pijnlijke herinnering aan hun dood? Als zowel hun aan- en afwezigheid een verwijzing naar genocide impliceren, wat is dan de verklarende waarde van hun visuele representatie? Op dezelfde manier refereert Calendar, waarin "geen enkele allusie wordt gemaakt op de Armeense genocide" volgens Rollet precies aan die genocide, terwijl een uitdrukkelijke vermelding ervan wellicht ook als bewijs van de aanwezigheid was beschouwd. Opnieuw stelt zich de vraag naar het verklarend potentieel van een stelling die lijkt te pretenderen alles, inclusief de contradicties, te kunnen verhelderen.

Niemand betwist de grote waarde van stiltes, van blanco's, van een ongedefinieerd gebaar in een film of een documentaire. Zelfs de door de wol geverfde menswetenschapper, die zich veilig waant achter zijn academische schermen van objectiviteit, wordt er ongetwijfeld op de meest onverwachte momenten, ongewild, door verrast en aangegrepen. Dat die ogenblikken waardevol zijn is zeker. Of ze erg veel verklaren (in de zin van het doorzien van betekenisvolle verbanden) is dat veel minder. Wellicht verdienen ze niet het verheven statuut dat Rollet hen verleent, al was het maar omdat zelfs de 'entre-images' hun betekenis deels ontlenen aan de 'images' zelf. Hoe dan ook stelt zich de vraag of het Niets geen al te mager alternatief vormt voor het - toegegeven, altijd slechts benaderende, deels onvolmaakte, nooit ten volle te (be)grijpen - Iets. 


\title{
Je suis une rémanence de l'histoire juive
}

\author{
Aharon Appelfeld
}

Par Julie Laval, Rédactrice - sites internet de théâtre

Ce texte ne se présente pas comme une analyse de l'ensemble de l'œuvre littéraire de Aharon Appelfeld. Il s'agira plutôt d'appréhender la question de son écriture et de son univers - mi-témoignage, mi-fiction - à travers le regard de la génération de la fin du XX $\mathrm{XX}^{\mathrm{e}}$ siècle, héritière de cette histoire, de cette mémoire.

\section{DU VÉCU À LA FICTION}

S'il y a bien eu un débat autour du roman Jan Karski de Yannick Haenel qui se proclame autant témoin (né en 1967 !) que les témoins survivants, brandissant l'argument de la fiction comme justification à toutes les interprétations possibles de l'Histoire, du passé, de la vérité, et ce, au nom du devoir de mémoire, on ne pourra que se questionner sur la manière dont nos générations devront et pourront témoigner. Seulement voilà : des témoins, il en reste ; et Appelfeld en est un. Témoin survivant, c'est ici le travail imaginatif et mémoriel qui nous intéresse et ce que sa littérature provoque chez des générations qui n’ont rien vu, rien vécu, juste entendu, prenant alors conscience qu'elles ne peuvent écrire sur la Shoah elle-même, mais uniquement sur sa transmission.

D’ailleurs, s'il fut catalogué par certains, à ses débuts, d'« écrivain de la Shoah », on peut reprendre ces mots de critiques qui nuancèrent cette qualification : «il n'écrit pas sur la Shoah, mais sur les marges de la Shoah. »

Appelfeld invente des histoires d'enfants confrontés à la cruauté des adultes dans un monde en proie à des démons. Et lorsqu'on a connu ces démons-là, on ne s'en remet pas, ils les poursuivent toute leur vie. Ils les poursuivent tellement que les déportés ne peuvent pas parler entre eux lorsqu'ils se retrouvent, ne peuvent pas communiquer leur souffrance, leur désespoir, parfois même ils s'évitent (Et la fureur ne s'est pas encore tue). Au-delà du traumatisme, Appelfeld écrit et crée sur la complexité des rapports humains, sur ce qu'on voudrait et qu'on ne peut exprimer, sur ce qu'on pourrait faire et qu'on ne peut appliquer.

Lauteur crée un genre nouveau. À la différence de Primo Levi, Elie Wiesel, Ruth Klüger, Buber Neumann, Appelfeld ne raconte pas « les camps». Il invente des histoires qui ne sont que la sienne propre, répétée à l'infini ; confrontant l'enfant qu'il était à la découverte d'un monde définitivement hostile, barbare, inhumain dans lequel il faut lutter chaque seconde. Plus qu'une lutte pour la survie, l'œuvre d'Appelfeld est une 
lutte pour la vie une fois que la mort l'a envahie ; ou : comment vivre après la mort ? Comment accueillir la lumière après les ténèbres ? Dans ce monde où se côtoient des figures aussi attachantes et des attitudes aussi condamnables, l'enfance est au cœur de son travail. Il témoigne de l'enfant qu'il était, racontant son histoire, s'appuyant sur sa mémoire, convoquant son imaginaire, tissant ainsi des possibilités d'expérience où la force de vie finit par l'emporter.

\section{LA FIN D'UN MONDE}

Appelfeld ne témoigne pas seulement de l'expérience traumatique. Il témoigne également de la fin d'un monde; de cette culture ashkénaze si particulière qu'on entretient de nos jours avec soin, qu'on transmet avec sérieux, mais qui n'existe plus. Les générations d'après n'ont cessé et ne cessent d'entretenir son passé, sa cuisine, sa littérature, transmettant aux enfants l'importance de s'inscrire dans une culture morte pour la faire revivre; communauté cosmopolite ou yiddishophone, des shtetls ou des capitales, assimilée ou communautaire, pratiquante ou laïque. Lécrivain est et incarne cette culture, il l'aime, il la porte, témoignant de l'horreur comme il témoigne de ce monde englouti. De ce monde qui existait et qui constituait un mélange de spiritualité et d'ouverture au monde, d'identité revendiquée et de discrétion assumée, d'histoire douloureuse et d'humour bien connu. Aussi, ce monde-là ne peut plus désormais exister que dans l'imaginaire de ceux à qui on l'a transmis et qui arrivent à le faire revivre à travers la création ou la fiction. Ce n'est pas témoigner pour ces générations, mais cela permet de ne pas oublier. Appelfeld, lui, conjugue les deux. C'est-à-dire qu'il fait revivre ce monde qu'il a connu à travers ses souvenirs, des sensations, des odeurs passées au travers d'histoires inventées, tout comme il témoigne de sa disparition.

Aussi, on peut se poser la question de savoir si le «témoignage » des générations d'après ne peut se faire - par la fiction - que sur l'évocation de ce monde d'AVANT. Quand Appelfeld dit « ce que j'écris, c'est une saga. Une saga sur la solitude. La solitude juive. Une longue, très longue solitude », il met déjà en effet en scène, par un procédé métaphorique, des enfants dont la toute première solitude qu'ils vivent est une différence qui n’appartient qu’à eux : Tsili est simple d'esprit, Bruno a un moignon. Appelfeld témoigne de ce monde « d'avant », mais également « d'après », c'est-à-dire de ses transformations.

\section{DU SHTETL À ISRAËL}

De cette rémanence de l'histoire juive, il a vu les changements, a vécu la tragédie, a observé les bouleversements pour, au final, ne pas vouloir cesser de témoigner de cette histoire. Il est le témoin d'un siècle. De sa Bucovine natale, où l'allemand était sa langue maternelle, il atterrira en Israël où l'hébreu était la langue officielle et où tout était à construire. Travail de la terre, travail de la langue, travail de la mémoire, histoire d'un témoin, qui, contrairement à une jeune personne de nos jours, se définit 


\section{LIBRAIRIE}

avant tout comme « un Juif qui écrit en Israël » et non comme un Israélien, pour montrer le déracinement perpétuel vécu, incarnant ainsi l'histoire d'un peuple en diaspora depuis plusieurs siècles. Terre de promesses sur la Terre promise, c'est en Israël qu'Appelfeld va construire une littérature qui n’appartient quà lui, qui témoigne tout en créant, donnant aux générations futures un regard particulier sur ce drame, obligeant ainsi les futurs « témoins » à bien réfléchir à ce qu'il faut dire avant d'écrire, à bien penser à ce qu'on veut faire avant d'agir. 